\title{
A NOVEL STRATEGY FOR EXPLORATION WITH MULTIPLE ROBOTS
}

\author{
Jonathan Rogge and Dirk Aeyels \\ SYSTeMS Research Group, Ghent University, Ghent, Belgium \\ Jonathan.Rogge@UGent.be,Dirk.Aeyels@UGent.be
}

Keywords: Multi-robot systems, coverage, exploration, demining.

\begin{abstract}
The present paper develops a novel strategy for the exploration of an unknown environment with a multirobot system. Contrary to most exploration problems, the topographical properties of the space need not be mapped. The algorithm we propose is inspired by methods used for complete coverage of an area, where all free space has to be physically covered by all robots. In the present paper it is required that the entire free space is covered by the sensors of the robots, with a certainty of $100 \%$. This weaker requirement enables us to scan more space in less time, compared to complete coverage algorithms. Moreover the shape of the robot formation adjusts itself to situations where obstacles, narrow spaces, etc. have to be passed. Communication between the robots is restricted to line-of-sight and to a maximum interdistance between robots. A direct application of the algorithm is mine field clearance.
\end{abstract}

\section{INTRODUCTION}

The research domain of multi-agent robot systems can be divided into subdomains according to the task given to the robot group (Ota, 2006). At present wellstudied subdomains are motion-planning (also called path-planning), formation-forming, region-sweeping, and combinations of the foregoing. The problem considered in the present paper belongs to the discipline comprising region-sweeping. In this discipline two different robot tasks are usually considered.

In the first task a group of robots receives the order to explore/map an unknown region. The goal is to obtain a detailed topography of the desired area. A typical approach to tackle the above problem with multiple robots assumes unlimited communication (Burgard et al., 2005): since exploration algorithms are already devised for a single robot it seems straightforward to divide the area to be explored into disjunct regions, each of which is assigned to a single robot. The robots communicate to each other the area they have explored so that no part of the free space will be explored twice unnecessarily. At no point during the task are the robots trying to form a fixed formation. Each robot explores a different part of the unknown region and sends its findings to a central device which combines the data received from the robots into one global map of the area.

Closely related to the exploring/mapping task is the second task, called complete coverage, where the robots have to move over all of the free surface in configuration space. Typical applications are mine field clearance, lawn mowing and snow cleaning. The coverage problem has been addressed in the literature both in a deterministic and a probabilistic setting. In the probabilistic approach the robots are considered as if they were fluid or gas molecules satisfying the appropriate physical laws of motion (Kerr et al., 2004), (Keymeulen and Decuyper, 1994). Just as a gas by diffusion fills an entire space, the robots will cover all free space when time tends to infinity. In the remainder of the paper we focus on the deterministic setting. In this setting the robot group typically forms (partial) formations to solve the task. Reference (Choset, 2001) gives a short overview of existing techniques for multi-robot coverage problems. Different approaches to the coverage problem are found in (Cortés et al., 2004), (Kurabayashi et al., 1996), (Wong and MacDonald, 2004), (Zheng et al., 2005) (Rekleitis et al., 2004) and (Kong et al., 2006). 
The problem statement of the present paper does not differ that much from the common exploration/mapping task and the complete coverage problem, but is rather a combination of both. It is required that all of the free space is sensed by the robots, but not necessarily physically covered. However, unlike the common exploration case, the sensing of the area does not have as goal to map the topography of the free space and the location of the obstacles in it. Our aim is to locate several unknown targets within the free space. Moreover, similar to the complete coverage setting we demand a $100 \%$ certainty that all free space has been covered by the sensors at the end of the exploration procedure, implying that all targets have been found. Since the robots no longer have to cover all free space physically, the novel algorithm will yield a time gain compared to complete coverage strategies. The developed algorithm is inspired by complete coverage strategies as (Rekleitis et al., 2004), (Kong et al., 2006). In these strategies it is assumed that the space to be explored does not have a maze-like structure with many narrow corridors, but is an open space containing only convex obstacles sparsely spread throughout. In the present paper we adopt these assumptions, postponing more complex geometries to future work. Our algorithm is presented in Section 2 of the paper. A short comparison between the algorithm presented here and that of (Rekleitis et al., 2004) is given in Section 3.

A specific application we have in mind is mine field clearance using chemical vapor microsensors (Gage, 1995). Once a landmine is deployed, the environment near the mine becomes contaminated with explosives derived from the charge contained in the mine. The vapor microsensors are able to detect the chemical vapor signature of the explosives emanating from the landmines from a distance. This implies that complete coverage algorithms may be too restrictive with respect to the demining problem. Performing the algorithm of the present paper, with the weaker requirement of sensor coverage, will result in a gain of time.

The algorithm can also be used in problems where a robot formation has to cross a terrain containing sparsely spread obstacles. There is a natural trade-off between coherence of the formation and avoidance of the obstacles. The robot group is allowed to split in order to pass the obstacles, resulting in faster progress of the group across the terrain. The algorithm ensures that once the obstacle is passed, the robots regroup.

\section{AN ALGORITHM FOR COMPLETE SENSOR COVERAGE}

\subsection{Setting}

Consider a population of $N$ identical robots. Each robot is equipped with two types of sensors. One type serves as a means to detect the goal targets to be found in the assigned area, e.g. landmines; the other type is used to detect and locate other robots and obstacles in the neighborhood of the robot ${ }^{1}$. Both sensors have a maximum detection range $s_{t}$ and $s_{r}$ respectively. It is assumed that targets which come within the radius of the corresponding sensor area $s_{t}$ or $s_{r}$ of the robot are always detected, and that if they are located farther away than the distance $s_{t}, s_{r}$ they are never detected. The robot configuration allows limited communication. First, this is expressed by the maximum detection range $s_{r}$ as described above. Second, lineof-sight communication is assumed: two robots can only sense each other if they are sufficiently close to each other and if there is no obstacle located on the straight line connecting both robots.

Two robots are called connected to each other when they sense each other. Every robot is assigned an index number. The initial state of the robot configuration is such that robot $i$ is connected to robots $i-1$ and $i+1, \forall i \in\{2, \ldots, N-1\}$. (Robot 1 is only connected to robot 2 and robot $N$ is only connected to robot $N-1$.) An example of such a configuration is depicted in Figure 1 for $N=6$. The dashed circles

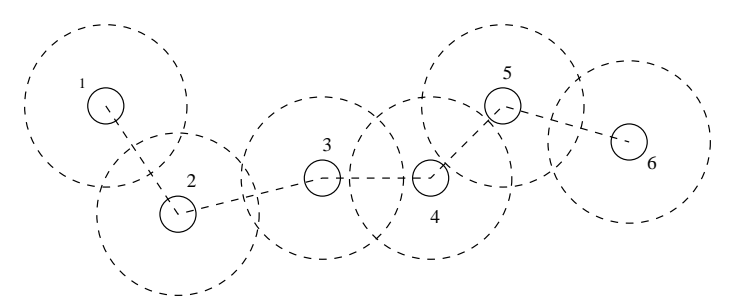

Figure 1: Overlapping sensor areas in a possible robot configuration.

have radius $s_{t}$ and signify the sensed area for goal targets of each robot. It is assumed that

$$
\frac{s_{r}}{2}<s_{t}<s_{r} .
$$

\footnotetext{
${ }^{1}$ In practice the latter type consists of two distinct minimally interfering IR-sensors: one sensing obstacles and the other sensing robots. Since this is not relevant for the theoretical description of the algorithm, these sensors are considered as if they are one and the same.
} 
The lower bound on $s_{t}$ in (1) ensures that the areas sensed for goal targets of neighboring robots partially overlap, as illustrated by Figure 1.

In a second step, we impose a preferred formation on the robot group as follows: each robot keeps a constant distance $d<s_{r}$ with its neighbors and observes them at preferred angles with respect to its forward direction. With notation from Figure 2, these angles are defined by:

$$
\begin{aligned}
& \alpha_{i}= \begin{cases}\pi / 4, & i \text { even, } \\
3 \pi / 4, & i \text { odd },\end{cases} \\
& \beta_{i}= \begin{cases}-\pi / 4, & i \text { even, } \\
-3 \pi / 4, & i \text { odd }\end{cases}
\end{aligned}
$$

Furthermore each robot is equipped with a compass. Together with the above defined angles, the forward direction of each robot (the same for all robots) is imposed at the initialization of the algorithm. The above conditions imply a robot formation with zigzag shape, as shown in Figure 3. Once the preferred formation is attained the scanning algorithm is started.

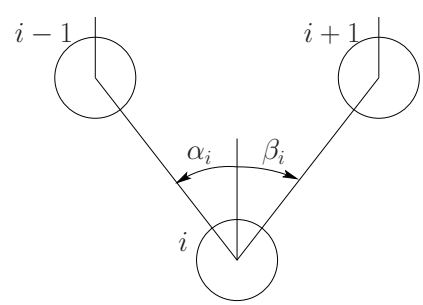

Figure 2: Defining the angles of the preferred robot configuration.

\subsection{The Scanning Algorithm}

Assume for simplicity that the area to be explored is a rectangular subset $S$ of $\mathbb{R}^{2}$. All obstacles contained in $S$ are assumed disjoint and convex. Divide the set $S$ into parallel (scanning) strips of width $\left(\frac{N}{2}-1\right) d$. This choice of the value of the width will be motivated later, in Section 2.4. Furthermore assume that $N$ and $d$ are such that all obstacles in $S$ have a diameter smaller than the width of a scanning strip. Fix the maximum allowed diameter at $\left(\frac{N}{2}-3\right) d$. The main idea of the algorithm is to let the group of robots sweep the area $S$ strip after strip in a zigzag-like pattern. Clearly, when there is a sufficient number of robots available the set $S$ can be regarded as one big strip, simplifying the algorithm since no transitions between consequent strips have to be performed.

In a first case we consider a strip where no objects are located on the boundary (see Figure 3). Robots 1 and $N$ are allocated the task to follow the boundaries of the strip at a constant distance at the constant velocity $v$. They can be considered leaders of the robot group. These two leader robots do not try to stay in the preferred formation, i.e. the condition on the corresponding angles $\alpha_{N}, \beta_{1}$ is removed, and they do not maintain a fixed interdistance $d$ with their neighbors. The remaining robots, however, still maintain the preferred formation. When no obstacles are present in the strip, the robots scan the strip for goal targets in the above defined preferred (rigid) formation moving at a velocity $v$.

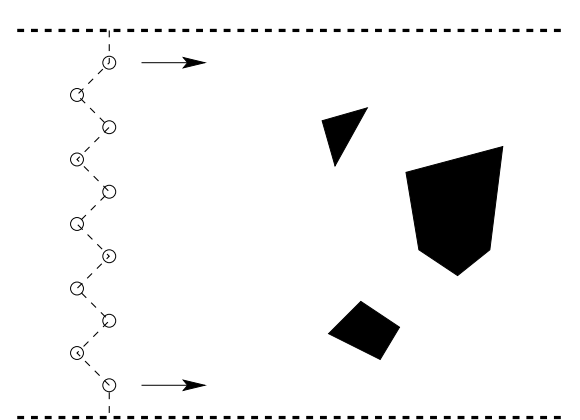

Figure 3: A depiction of the algorithm. The arrows indicate the constant velocity of both leader robots. The dashed lines represent the strip boundaries.

When an obstacle is encountered the algorithm aims to guide the robot group past it in a time-optimal way. The leader robots start driving at a preset velocity $v_{0}<v$ (see Section 3 ); the group is split into two subgroups in order to move around the obstacle. The subgroups rejoin after passing the obstacle to resume the preferred formation structure.

Assume for reasons of simplicity that the number of robots $N$ is even. Consider the situation where robot $m$ encounters an object on its path such that it cannot stay in the preferred formation any longer. More precisely, the sensors of robot $m$ measure

- an interdistance between the obstacle and the robot smaller than a preset distance $d_{o}<s_{r}$,

- the position of the obstacle at an angle with its forward direction inside the interval $(-\gamma, \gamma)$, with $\gamma$ a fixed value inside the interval $\left(0, \frac{\pi}{4}\right)$.

The presence of the obstacle is communicated to all the robots in the group. Each robot takes on a different role such that two subgroups will be formed. The robots with index $i \in S_{1}:=\{2, \ldots, N / 2\}$ now follow the neighboring robot with corresponding index $i-1$. Similarly, robots with index $i \in S_{2}:=$ $\{N / 2+1, \ldots, N-1\}$ follow the neighboring robot with index $i+1$. More precisely, the robot with in- 

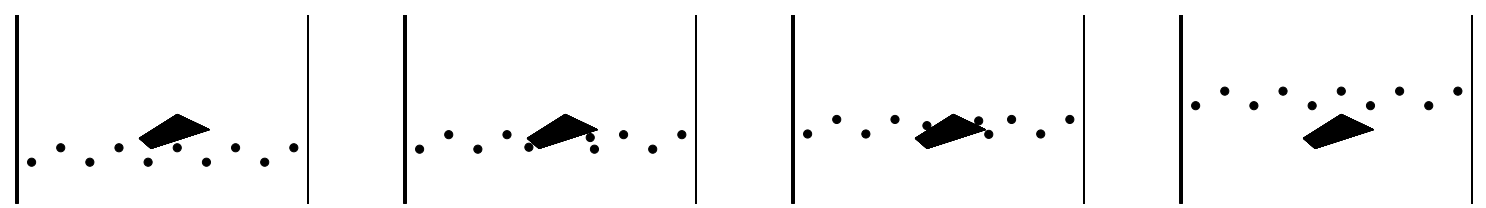

Figure 4: A group of 10 robots passing an obstacle. Only robots 5, 6 and 7 apply wall-following around the obstacle during the maneuver. This is shown in the second and third picture. The first picture shows the formation at the moment of encountering the obstacle. The fourth picture presents the formation after having completely past the obstacle.

dex $i$ tries to reach the following coordinates:

$$
\begin{cases}\left(x_{i-1}+d \sin \frac{\pi}{4}, y_{i-1}+(-1)^{i} d \cos \frac{\pi}{4}\right), & \text { if } i \in S_{1}, \\ \left(x_{i+1}-d \sin \frac{\pi}{4} d, y_{i+1}+(-1)^{i} d \cos \frac{\pi}{4}\right), & \text { if } i \in S_{2} .\end{cases}
$$

These coordinates are considered with respect to a right-handed $(x, y)$-frame with the $y$-axis parallel to the strip boundary, and directed into the driving direction of the leader robots. Each robot still tries to stay in the preferred formation, but in order to do so only takes information of one neighbor into account. Moreover, the condition on the relative position between the neighboring robots $N / 2$ and $N / 2+1$ is suspended, which will lead to the splitting of the robot group. Notice that indifferent of the robot that observes the obstacle first, the group will split between robots $N / 2$ and $N / 2+1$. This choice is motivated in Section 2.4.

Consider the situation for robot $i$ where one of the following occurs:

- The desired position (3) cannot be reached,

- The obstacle is blocking the straight path between the present position of robot $i$ and its desired position,

- Robot $i$ does not detect its neighbor necessary to determine its preferred position.

If this situation occurs, the robot receives the order to follow the edge of the obstacle, keeping the obstacle on its right if $i \in S_{1}$, or its left if $i \in S_{2}$. This behavior is called wall-following. The robot continues to wall-follow around the obstacle until none of the above conditions is satisfied. After that, it assumes its desired position again. If all robots have past the obstacle, each robot is again able to reach its desired position in the preferred formation. In particular, robots $N / 2$ and $N / 2+1$ will meet again in their desired relative position. When this happens a signal is sent to all robots with the message that the group has past the obstacle.

A simulation of the above described algorithm is presented in Figure 4 with $N=10$ and $m=6$.
Remark. It may occur that a robot cannot reach its desired position because it is located too far away from its present position. Then the robot simply rides towards the desired position at the maximum allowed velocity, trying to catch up.

Remark. If the number of robots $N^{*}$ in the (sub)group is not even, then the indices of the robots where the robot group splits are $\left\lfloor N^{*} / 2\right\rfloor$ and $\left[N^{*} / 2\right\rceil$, where $\lfloor$.$\rfloor is the function giving the largest integer less$ than or equal to its argument, and similarly, $\lceil$.$\rceil gives$ the smallest integer greater than or equal to its argument.

\subsection{Multiple Obstacles}

Suppose the robot group is already split into two subgroups and a robot in one of the subgroups encounters a second obstacle. The above obstacle avoidance algorithm can be made modular in order to solve this problem. A group can only split if both robots at the extremities of the group are leader robots, similar to the initial configuration. Assume group $S_{1}$ encounters a second obstacle. Robot $N / 2$ is then turned into a leader robot. Instead of following a strip boundary it is ordered to follow the edge of the first obstacle, until it meets its neighbor $N / 2+1$ or until group $S_{1}$ has past the second obstacle. In the latter case, robot $N / 2$ takes on its role as a follower of robot $N / 2-1$ again, in the former case it turns into a follower of $N / 2+1$. The group $S_{1}$ is split into the middle and the algorithm described in the previous section is performed with leader robots 1 and $N / 2$. In order for each robot to know which role to assume, it keeps track of how many times its subgroup is split.

Clearly, the number of times this splitting can be repeated is limited. We require a subgroup to consist of at least 3 robots: two leader robots on each side of the group, plus at least one robot in the middle attempting to follow both leaders while maintaining the formation structure. The middle robot ensures that the 
discs of sensed area of the separate robots overlap for all time instants.

\subsection{Adaptation of the Basic Algorithm}

Consider a worst case scenario as sketched in Figure 5. The robot formation splits into two subgroups, and the group on the left hand side moves through the gap between the obstacle and the left boundary of the scanning strip. Once past the gap the robots in this subgroup have to spread out, since the distance between the obstacle and the left boundary increases and we want to sense all of free space between the boundary and the obstacle. The obstacle has such a shape that the robots have to spread out across almost the entire width of the scanning strip before meeting a robot member of the right subgroup. The basic algorithm is modified as follows. When robot $N / 2$ (resp. $N / 2+1$ ) encounters the obstacle, it is now programmed to follow the obstacle's edge until it meets its neighbor $N / 2+1$ (resp. $N / 2$ ). Additionally, it ensures that its neighbor $N / 2-1$ (resp. $N / 2+1$ ) stays in its detection range by sending a signal to the other robots of its subgroup to increase the angle $\pi / 4$ of (3). This changes the desired position of each robot in the subgroup resulting in a stretching out of the group, as far as necessary. The above modified algorithm

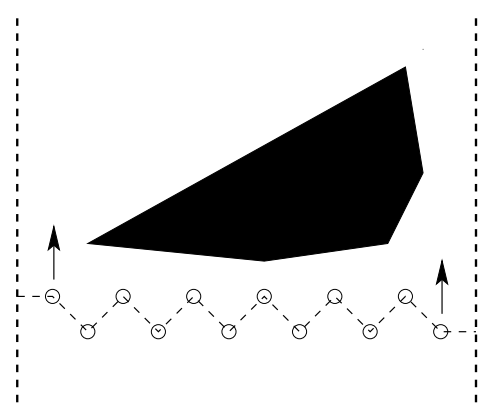

Figure 5: A depiction of the worst case scenario in the algorithm.

justifies our choice of initial formation and width of the scanning strip. If we had naively chosen a value $(N-1) d$ as the width of a scanning strip, the initial preferred robot formation would be able to span this entire distance, namely by forming a line with the angles defined in Section 2.1 equal to $\alpha_{i}=-\beta_{i}=\pi / 2$. However, one subgroup, consisting of only half of the number of robots, would not be able to span this distance, resulting in either an error message from the algorithm or in unscanned areas, if a situation described in Figure 5 was encountered.

Closely related to this observation is the choice to split the robot group precisely in the middle. Since the sensor range of each robot is limited and the robots operate in an unknown environment, the shape of each obstacle is unknown. To guarantee that the area around the obstacle is fully covered by the sensors, we have to supply a sufficient number of robots to both sides of the obstacle. For instance, when the shape of the obstacle in Figure 5 is known a priori, one can decide to send more than half of the robots to the left of the obstacle. Consider the case where the obstacle is reflected with respect to the vertical axis. In this case sending less than half of the robots to the right would lead to uncovered areas or an error message in the algorithm. With limited sensor information it is not possible to discriminate between the situation of Figure 5 and its reflected version. This leads us to always split the group into two equal parts.

An alternative solution for this problem could be a more intelligent algorithm where individual robots transmit more sensor data to the others in order to find possible bounds on the size of the encountered obstacle. If the obstacle is small, a better way to split is right at the place where the obstacle is encountered. The robots do not have to deviate much from the preferred formation, which decreases the probability on error messages from deforming the robot configuration. This idea may be incorporated in future work.

\subsection{Obstacles Located on the Boundary between Two Strips}

Throughout the paper the obstacles are assumed to have a convex shape, in order to avoid robot groups getting stuck in a dead end. However, there is one case of dead ends we cannot avoid by the above assumption. A dead end can occur when an obstacle is located on the boundary between two strips, as presented on the left hand side of Figure 6 . Since the robots have limited sensor information, they cannot conclude a priori whether an encountered obstacle stretches out into a neighboring strip or not. We are forced to let the algorithm run until a dead end is observed.

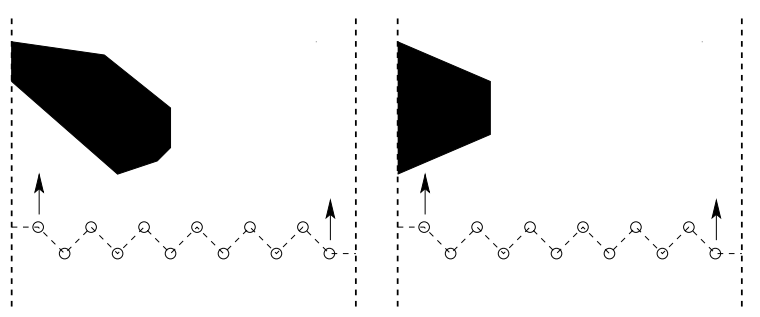

Figure 6: Two situations where an obstacle is located on the boundary between strips. On the left hand side a dead end situation arises; on the right hand side one of the leader robots guides the group around the obstacle. 
Before tackling the dead end problem, let us treat the case presented on the right hand side of Figure 6, which does not lead to a dead end situation. Consider an $(x, y)$-frame with the $y$-axis parallel to the strip boundary, and directed into the driving direction of the leader robots. When the leader robot encounters the obstacle, the algorithm assigns to this leader a wall-following procedure around the obstacle. The leader keeps the obstacle either on its right or left (depending on its position in the robot formation) while moving into the interior of the strip away from the strip boundary. As can be concluded from the picture, the $y$-coordinate of the leader increases while moving around the obstacle. We wish to keep the velocity component of the leader robot parallel to the strip boundary equal to $v$. Since the robot deviates from its straight path parallel to the strip boundary, this implies it has to speed up. When the leader reaches the strip boundary again, it switches back to the original task of moving parallel to the boundary.

Now consider the left hand side of Figure 6. A dead end is detected by the algorithm when two conditions are satisfied:

- one of the leader robots cannot move into the desired direction parallel to the strip boundary, because an obstacle is blocking the way.

- when the leader robot starts wall-following the obstacle as described above, the value of its $y$ coordinate decreases.

As soon as a dead end is observed by the leader robot, it changes its behavior and stops its wall following algorithm. Instead, it projects its corresponding strip boundary $(N / 2-1) d / 8$ units outwards and resumes the original scanning algorithm with respect to the new boundary. If the extra width turns out to be insufficient to guide the robot subgroup around the obstacle outside of the original scanning strip, the boundary is projected a second (third,...) time. This way the subgroup which was stuck in the dead end is guided around the obstacle. When both subgroups reestablish contact, the leader robot returns to the original strip boundary. This behavior is faster and easier to implement than a turning-back scenario, where the subgroup of robots which meets a dead end retraces it steps to go around the obstacle inside the original scanning strip.

Remark. The above situation with a solid wall as strip boundary, forcing a turning-back maneuver, is precluded.

\subsection{The Transition from One Strip to the Next}

When the robot group reaches the end of a scanning strip, it needs to be transported to the next strip. This is done in a few easy steps. Consider the situation of Figure 7. First the right leader changes its behavior into that of a robot in the interior of the formation, i.e. it tries to attain the desired formation. The left leader moves $(N / 2-1) d$ units to the left perpendicular to the strip boundary. The rightmost robot resumes its leader role and all robots reverse their forward direction with respect to the desired direction in the previous strip. Naturally, every time the end of a strip is reached, the roles of left and right leader alternate, so that the robot group does not get trapped into a loop consisting of two strips.

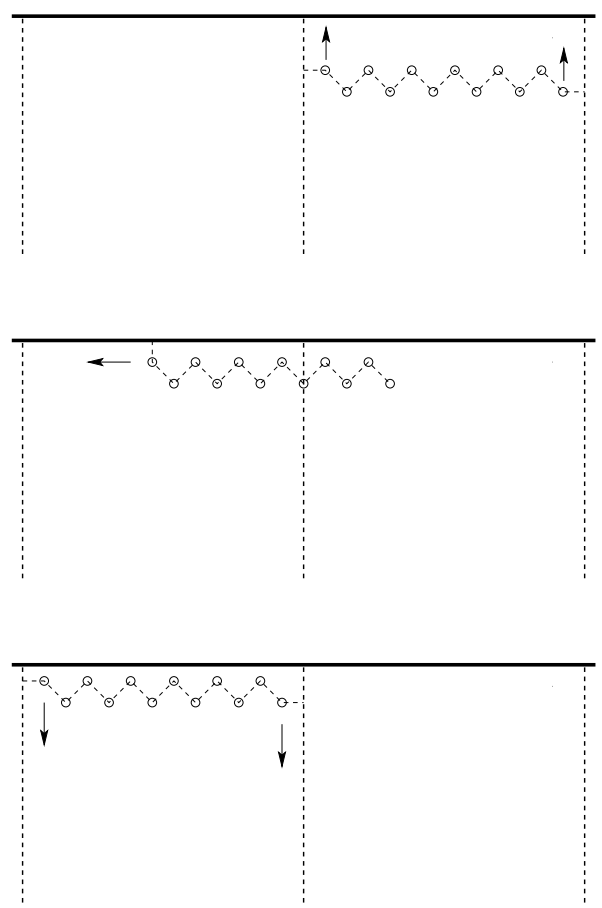

Figure 7: The robot group moves from the end of a scanning strip to the start of the next strip.

\section{COMPARISON WITH COMPLETE COVERAGE ALGORITHMS}

The algorithm presented in this paper is inspired by the complete coverage algorithm presented in (Rekleitis et al., 2004), which is depicted in Figure 8. The authors of (Rekleitis et al., 2004) propose the follow- 
ing robot configuration: 2 leader robots, following the strip boundaries, and a number of interior robots, traveling back and forth between the strip boundaries physically covering all the free space. Contrary to the algorithm proposed in the present paper, the leader robots maintain line-of-sight contact between each other. When an obstacle appears between the two leaders the line-of-sight contact is lost and the obstacle is detected. An appropriate control action is then taken by splitting the platoon and the algorithm is repeated on both sides of the obstacle. The splitting procedure includes the creation of two extra leader robots, as shown in Figure 8. Remark that the leaders are allowed to move ahead of the rest of the robot group and hence group coherence is not maintained or desired, contrary to our approach.

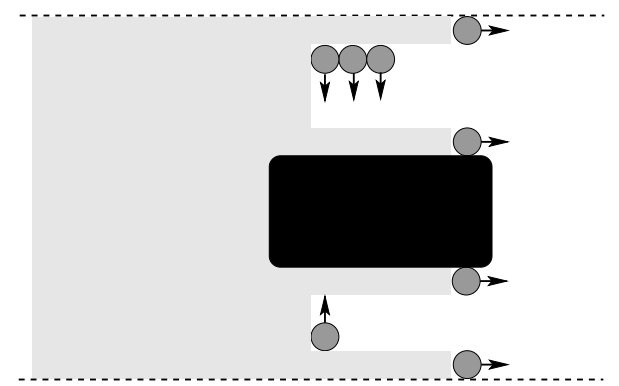

Figure 8: A depiction of the complete coverage algorithm by Rekleitis et al.

In the remainder of this section we will compare speed of performance of the present algorithm with the algorithm of (Rekleitis et al., 2004). In order to do so, realistic distance values are considered. Chemical vapor sensors detecting mines have a range $s_{t}=$ $1.70 \mathrm{~m}$. Obstacles and other robots can be detected by laser based sensors with a range of $s_{r}=3.3 \mathrm{~m}$ such that (1) is satisfied. Assume the robots themselves possess a diameter of $0.3 \mathrm{~m}$ and set the fixed interdistance $d$ between neighboring robots in the preferred formation equal to $s_{r}$. With $N$ the number of robots in the group, this yields a strip width of $1.65(N-2) \mathrm{m}$.

When no obstacles are encountered, the robots are allowed to move at a preset maximum velocity $v_{\max }$. In the algorithm of the present paper $v_{\max }$ is directed parallel to the strip boundary, whereas the interior robots in (Rekleitis et al., 2004) travel back and forth inside the strip at $v_{\max }$. It can be proven that for the latter case with the values given above the speed of progress parallel to the strip boundary is $v_{\max } / 6$.

In the presence of obstacles a comparison is more difficult. First consider the complete coverage algorithm (Rekleitis et al., 2004). As can be concluded from Figure 8 , in the presence of an obstacle the robots will advance faster parallel to the strip bound- ary, since the space occupied by the obstacle does not have to be covered. The robot group will proceed fastest when the shape of the obstacle is such that there is no space left for the robots to travel back and forth between obstacle and strip boundary. Hence, depending on size and shape of the obstacle the robots advance with a speed between $v_{\max } / 6$ and $v_{\max }$. Now, consider the algorithm of the present paper. Some interior robots perform wall-following around the obstacles. This implies their path is longer than the path of the leader robots. If the leader robots keep moving at the maximum allowed velocity, those interior robots will never again be able to reach their desired position inside the formation after the obstacle is past. Hence, when an obstacle is encountered the leaders have to take on a velocity $v_{0}$ which is smaller than $v_{\max }$. This velocity $v_{0}$ is determined as follows. The middle robots $N / 2$ and $N / 2+1$ transmit their positions via the other robots to their respective leader robots. The leaders adjust their velocity $v_{0}$ such that the difference between their $y$-coordinate and the $y$ coordinate of the corresponding $\operatorname{robot} N / 2$ or $N / 2+1$ stays at all time within a prespecified bound. The middle robots only slow down the group significantly during the first and last stage of their obstacle following, i.e. when moving away from or towards the strip boundary without significantly advancing parallel to it. As soon as there is enough free space ahead of the middle robots, the subgroup is again allowed to move parallel to the strip boundary with a speed close to $v_{\max }$.

From the above observations the following is concluded. The robot group in the present algorithm slows down to pass an obstacle, but for most of the time the speed will be close to $v_{\max }$. The robot group of the complete coverage algorithm speeds up when passing an obstacle, but for most obstacles the algorithm still requires a robot group moving back and forth between the obstacle and the strip boundary. This implies that the increased speed will on average be closer to $v_{\max } / 6$ than to $v_{\max }$. Hence, in generic cases, the present algorithm performs faster than the complete coverage strategy even in the presence of obstacles.

\section{CONCLUSIONS}

The present paper described a novel strategy for multi-robot exploration of an unknown environment with guarantee of total sensor coverage. The algorithm we proposed is inspired by methods used for complete coverage as described in (Rekleitis et al., 2004). We took advantage of the fact that only com- 
plete sensor coverage is required. We let the robots form a spring-like formation which scans the area in strips. In the presence of obstacles the formation is deformed and split in two in order to circumvent the obstacles and to adapt to the varying width of the free space.

\section{ACKNOWLEDGEMENTS}

This paper presents research results of the Belgian Programme on Interuniversity Attraction Poles, initiated by the Belgian Federal Science Policy Office. The scientific responsibility rests with its authors.

\section{REFERENCES}

Burgard, W., Moors, M., Stachniss, C., and Schneider, F. (2005). Coordinated multi-robot exploration. IEEE Transactions on Robotics, 21(3):376-386.

Choset, H. (2001). Coverage for robotics - a survey of recent results. Annals of Mathematics and Artificial Intelligence, 31:113-126.

Cortés, J., Martínez, S., Karatas, T., and Bullo, F. (2004). Coverage control for mobile sensing networks. IEEE Transactions on Robotics and Automation, 20(2):243255.

Gage, D. (1995). Many-robots mcm search systems. In Proceedings of Autonomous Vehicles in Mine Countermeasures Symposium.

Kerr, W., Spears, D., Spears, W., and Thayer, D. (2004). Two formal gas models for multi-agent sweeping and obstacle avoidance. In Formal Approaches to AgentBased Systems, Third International Workshop, pages 111-130.

Keymeulen, D. and Decuyper, J. (1994). The fluid dynamics applied to mobile robot motion: the stream field method. In Proceedings of 1994 IEEE International Conference on Robotics and Automation, pages 378385, Piscataway, NJ, USA.

Kong, C. S., Peng, N. A., and Rekleitis, I. (2006). Distributed coverage with multi-robot system. In Proceedings of 2006 IEEE International Conference on Robotics and Automation, pages 2423-2429, Orlando, Florida, USA.

Kurabayashi, D., Ota, J., Arai, T., and Yosada, E. (1996). Cooperative sweeping by multiple robots. In Proc. 1996 IEEE International Conference on Robotics and Automation.

Ota, J. (2006). Multi-agent robot systems as distributed autonomous systems. Advanced engineering informatics, 20:59- 70 .

Rekleitis, I., Lee-Shue, V., New, A. P., and Choset, H. (2004). Limited communication, multi-robot team based coverage. In Proc. 2004 IEEE International Conference on Robotics and Automation.
Wong, S. and MacDonald, B. (2004). Complete coverage by mobile robots using slice decomposition based on natural landmarks. In Proc. Eighth Pacific Rim International conference on Artificial Intelligence. Lecture Notes in Artificial Intelligence., volume 3157, pages 683-692.

Zheng, X., Jain, S., Koenig, S., and Kempe, D. (2005). Multi-robot forest coverage. In Proceedings of the IEEE International Conference on Intelligent Robots and Systems. 